\title{
A NEW HIERARCHY OF INTEGRABLE SYSTEM OF $1+2$ DIMENSIONS: FROM NEWTON'S LAW TO GENERALIZED HAMILTONIAN SYSTEM. PART II
}

\author{
XUNCHENG HUANG AND GUIZHANG TU
}

Received 24 November 2004; Revised 11 December 2005; Accepted 18 December 2005

The Hamiltonian equation provides us an alternate description of the basic physical laws of motion, which is used to be described by Newton's law. The research on Hamiltonian integrable systems is one of the most important topics in the theory of solitons. This article proposes a new hierarchy of integrable systems of $1+2$ dimensions with its Hamiltonian form by following the residue approach of Fokas and Tu. The new hierarchy of integrable system is of fundamental interest in studying the Hamiltonian systems.

Copyright (c) 2006 Hindawi Publishing Corporation. All rights reserved.

\section{Introduction}

The classical theory of mechanics began with the fundamental Newton's laws of motion. The second law asserts that the rate of change of the momentum of a body is proportional to the resultant external force that acts on the body. With an appropriate choice of physical units and the assumption that the mass stays constant, we may express that law in the familiar form:

$$
\text { mass } \times \text { acceleration }=\text { force } \text { or } m a=F,
$$

where $m$ and $a$ represent the mass and acceleration of the body and $F$ is the external force. Suppose that the motion takes place in the 3 -dimensional Euclidean space $R^{3}$, then in Cartesian coordinates the equation becomes

$$
m \frac{d^{2} r}{d t^{2}}=F
$$

where $r \equiv(x, y, z)$ is the position of the body and $F \equiv(X, Y, Z)$ is the force.

If we consider a system consisting of $N$ particles, then the corresponding equation of motion becomes

$$
m_{i} \frac{d^{2} r_{i}}{d t^{2}}=F_{i} \quad(i=1,2, \ldots, N)
$$

Hindawi Publishing Corporation

International Journal of Mathematics and Mathematical Sciences

Volume 2006, Article ID 70747, Pages 1-10

DOI 10.1155/IJMMS/2006/70747 
In many cases there exist geometrical constraints, which limit the motion of the system. For example, the motion may be along a surface. In the presence of constraints the coordinates $r_{i}=\left(x_{i}, y_{i}, c_{i}\right),(i=1,2, \ldots, n)$ are no longer all independent and are usually connected by $k$ equations: $G_{j}(x, y, z)=0,(j=1,2, \ldots, k)$. Thus, only $n=3 N-k$ coordinates are independent; in this case it is more convenient and natural to use the generalized coordinates $q_{1}, q_{2}, \ldots, q_{n}$ so that we have

$$
r_{i}=r_{i}\left(q_{1}, \ldots, q_{n}\right) \quad(i=1,2, \ldots, N)
$$

or, in terms of generalized coordinates,

$$
\frac{d}{d t}\left(\frac{\partial T}{\partial \dot{q}_{j}}\right)-\frac{\partial T}{\partial q_{j}}=Q_{j} \quad(j=1,2, \ldots, n),
$$

where $Q_{j}=\Sigma_{i} F_{i} \cdot\left(\partial r_{i} / \partial q_{j}\right)$ is the so-called generalized force, and

$$
T=\sum_{i} \frac{1}{2} m_{i}\left(\dot{r}_{i} \cdot \dot{r}_{i}\right)=\sum_{i} \frac{1}{2} m_{i}\left(\dot{x}_{i}^{2}+\dot{y}_{i}^{2}+\dot{z}_{i}^{2}\right)
$$

stands for the kinetic energy of the system.

If the system is conservative, then the force $F_{i}$ can be expressed in terms of a scalar potential function $V=V(x)$, called the potential energy, in the form

$$
\begin{gathered}
F_{i}=-\nabla_{i} V \equiv-\left(\frac{\partial V}{\partial x_{i}}, \frac{\partial V}{\partial y_{i}}, \frac{\partial V}{\partial z_{i}}\right) \quad(i=1,2, \ldots, N), \\
Q_{j}=\sum F_{i} \cdot \frac{\partial r_{i}}{\partial q_{i}}=-\sum \nabla_{i} V \cdot \frac{\partial r_{i}}{\partial q_{j}}=-\frac{\partial V}{\partial q_{j}} \quad(j=1,2, \ldots, n) .
\end{gathered}
$$

Substituting the above equation into (1.5), we obtain the following equation of motion for a conservative system:

$$
\frac{d}{d t}\left(\frac{\partial L}{\partial \dot{q}_{j}}\right)-\frac{\partial L}{\partial q_{j}}=0 \quad(j=1,2, \ldots, n),
$$

where

$$
L=T-V
$$

We call (1.8) Lagrange's equation of motion and $L$ the Lagrangian of the system.

From the above derivation it is clear that $T=T(q, \dot{q}), V=V(q)$ and that Lagrange's equation (1.8) is a set of ordinary differential equations of the second order.

A further important development in classical mechanics consists in reformulating (1.8) as a set of $2 n$ ordinary differential equations of the first order. This can be done by introducing the generalized momenta $p_{i}$,

$$
p_{i}=\frac{\partial L}{\partial \dot{q}_{i}} \quad(i=1,2, \ldots, n)
$$


and the Hamiltonian $H$,

$$
H(p, q)=\sum_{i=1}^{n} \dot{q}_{i} p_{i}-L(q, \dot{q}),
$$

where, for simplicity, we assume that both $H$ and $L$ are not explicitly dependent on $t$. Then, on the one hand, we have

$$
\begin{gathered}
d H \equiv d H(p, q)=\sum \frac{\partial H}{\partial q_{i}} d q_{i}+\sum \frac{\partial H}{\partial p_{i}} d p_{i} \\
d H=d\left(\sum_{i=1}^{n} \dot{q}_{i} p_{i}-L(q, \dot{q})\right)=\sum \dot{q}_{i} d p_{i}+\sum p_{i} d \dot{q}_{i}-\sum \frac{\partial L}{\partial \dot{q}_{i}} d \dot{q}_{i}-\sum \frac{\partial L}{\partial q_{i}} d q_{i} .
\end{gathered}
$$

Then, by (1.10) and (1.8), we have $\partial L / \partial q_{i}=\dot{p}_{i}$, and thus

$$
d H=\sum \dot{q}_{i} d p_{i}-\sum \dot{p}_{i d q_{i}}
$$

Comparing the above equation with (1.12), we deduce the celebrated canonical equation of Hamilton for $q_{i}$ and $p_{i}$,

$$
\dot{q}_{i}=\frac{\partial H}{\partial p_{i}}, \quad \dot{p}_{i}=-\frac{\partial H}{\partial q_{i}} \quad(i=1,2, \ldots, n) .
$$

The above Hamiltonian equation provides us with an alternate description of the basic physical laws of motion. In this description, the coordinates $q_{i}$ and the momenta $p_{i}$ take on equal status: both are treated as independent variables and appear in the equation in a symmetric manner. This equal status gives us the freedom to choose the independent variables as desired, which is convenient in many circumstances. However, the real advantage of writing the equations of motion in their Hamiltonian form lies in the fact that it gives us a deeper insight into the nature of physical laws. Thus, modern theory of mechanics to be based on symplectic geometry and also formed into the KAM theorem concerning the stability of motion represents an important contribution to physics. From a physical point of view, the Hamiltonian formulation provides us with a most suitable mathematical framework for the study of statistical mechanics and quantum mechanics. Indeed, the development of these two areas was based entirely on Hamiltonian mechanics.

Nowadays, the theory of Hamiltonian systems has developed into one of the richest areas of mathematical physics. The study of these systems involves many branches of physics as well as mathematics, ranging from fluid mechanics to quantum mechanics, from classical theory of differential equations to modern representation theory of Lie groups. Yet, the power and beauty of this theory have not been fully recognized and it still inspires new ideas and provides a springboard for new developments.

One remarkable development of the theory of the classical Hamiltonian systems is the successful extension from finite-dimensional to infinite-dimensional systems. To describe 
this development, let us reformulate the Hamiltonian equation (1.15) into a form that is convenient to make such an extension. Thus, we introduce the vectors

$$
\begin{gathered}
u=\left(q_{1}, \ldots, q_{n}, p_{1}, \ldots, p_{n}\right)^{T} \\
\nabla H=\left(\frac{\partial H}{\partial q_{i}}, \ldots, \frac{\partial H}{\partial q_{n}}, \frac{\partial H}{\partial p_{1}}, \ldots, \frac{\partial H}{\partial p_{n}}\right)^{T}
\end{gathered}
$$

and the matrix

$$
J=\left[\begin{array}{cc}
0 & I \\
-I & 0
\end{array}\right]
$$

where $I$ stands for the $n \times n$ identity matrix. The Hamiltonian equation (1.15) can now be expressed as

$$
u_{i}=J \nabla H
$$

and the classical Poisson bracket

$$
\{f, g\}=\sum_{i=1}^{n}\left(\frac{\partial f}{\partial p_{i}} \frac{\partial g}{\partial q_{i}}-\frac{\partial f}{\partial q_{i}} \frac{\partial g}{\partial q_{i}}\right)
$$

can be written as

$$
\{f, g\}=(J \nabla f, \nabla g)
$$

where $(F, G)=\sum F_{i} G_{i}$ denotes the usual inner product of vectors $F=\left(F_{i}\right)$ and $G=\left(G_{i}\right)$. As a result of this reformulation of the classical Hamiltonian equation we have arrived at a wider class of equation, the so-called generalized Hamiltonian equations. These equations are usually partial differential equations that may include also some difference, difference-differential and integro-differential equations. Therefore, they are capable of modeling a variety of infinite-dimensional physical phenomena. In this extended case, the Hamiltonian function $H$ should be replaced by a Hamiltonian functional $H$, and its gradient $\nabla H$ is then defined by

$$
(\nabla H(u), v)=\left.\frac{d}{d \varepsilon}\right|_{\varepsilon=0} H(u+\varepsilon v) .
$$

Moreover, the matrix $J$ that appeared originally in (1.18) has to be replaced by a linear operator, which we call a Hamiltonian operator. To be more precise, a linear operator $J$ is called a Hamiltonian operator if the corresponding Poisson bracket (1.20) gives a Lie algebraic structure on a set of functionals, that is, if the following is true:

$$
\begin{gathered}
\{f, g\}=-\{g, f\}, \\
\{\alpha f+\beta g, h\}=\alpha\{f, h\}+\beta\{g, h\} \quad(\alpha, \beta=\text { const }), \\
\{f,\{g, h\}\}+\{g,\{h, f\}\}+\{h\{f, g\}\}=0 .
\end{gathered}
$$


The most important example of such a generalized Hamiltonian system is the celebrated korteweg-de Vries (KdV) equation:

$$
u_{t}=u_{x x x}+6 u u_{x}
$$

which describes the motion of shallow water. Setting $J=D=\partial / \partial x$, we can rewrite the $\mathrm{KdV}$ equation in the form

$$
u_{t}=\frac{\partial}{\partial x}\left(u_{x x}+3 u^{2}\right)=D \frac{\delta}{\delta u}\left(u^{3}-\frac{1}{4} u_{x}^{2}\right)
$$

where $\delta / \delta u=\sum_{n \geq 0}(-1)^{i}\left(d^{n} / d x^{n}\right)\left(\partial / \partial u^{n}\right)$ represents the variational derivative, and $d / d x=D+\sum_{n \geq 1} u^{n}\left(\partial / \partial u^{(n-1)}\right), u^{(n)}=D^{n} u$. It will be shown later that $J=\partial$ is indeed a Hamiltonian operator. Thus, the KdV equation serves as a typical example of a generalized Hamiltonian system.

One of the basic theorems in classical mechanics is the Liouville theorem. It states that if one can find a set of $n$ independent functions $f_{i}=f_{i}(p, q),(i=q, \ldots, n)$, which are the first integrals of the Hamiltonian system (1.15) and are involution in pairs: $\left\{f_{i}, f_{j}\right\}=0$, $1 \leq i, j \leq n$, then one can find a canonical transformation $(p, q) \rightarrow(P, Q)$, such that the original equation (1.15) takes the simple form

$$
\dot{Q}_{i}=\frac{\partial \bar{H}}{\partial P_{i}} \equiv 0, \quad \dot{P}_{i}=\frac{\partial \bar{H}}{\partial Q_{i}},
$$

where $\bar{H}(Q, P) \equiv H(p(Q, P), q(Q, P))$. The above equation can be easily integrated to obtain

$$
Q_{i} \equiv \alpha_{i}, \quad P_{i}=\omega_{i} t+\omega_{0 i}
$$

where $\alpha_{i}, \omega_{i}$, and $\omega_{0 i}$ are constants, and

$$
\omega_{i}=\frac{\partial \bar{H}(Q)}{\partial Q_{i}}=\text { const. }
$$

The set of variables $Q_{i}$ and $P_{i}(i=1, \ldots, n)$ are usually called the action-angle variables. The Liouville theorem or its modern version, the Liouville-Arnold theorem, indicates an effective way to find the solutions of the above system. In deference to this important theorem we define a generalized Hamiltonian equation to be Liouville integrable if it has an infinite number of independent first integrals, which are in involution in pairs. Though this definition on integrability is rather formal in the sense that it does not indicate a clear way on how to integrate the system explicitly, there is strong evidence to suggest that in many important cases it is possible to find some analytical solutions to these equations, and to find an infinite number of variables that look like action-angle variables. Nowadays, the theory of integrable Hamiltonian systems has developed into one of the most fruitful research areas, and the theoretical and methological progress made in the past 
twenty years has been recognized as one of the most remarkable achievements in the area of applied mathematics.

We have shown that the KdV equation can be put in the form of generalized Hamiltonian equation. Having done this, it is natural to ask the following questions.

(1) Is KdV equation integrable in the sense of Liouville?

(2) For a given nonlinear equation, especially for those equations that are widely used in various branches of physics, how can we judge whether the equation is Liouville integrable?

(3) How can we search for new integrable Hamiltonian systems?

(4) Since we have reason to believe that these integrable systems have some common algebraic and geometrical structure and share some special features, what is the structure and what are these special features?

To summarize, we see that to put the KdV equation into its Hamiltonian form is not the end but just the beginning of many interesting topics that we will consider. We will come back to each of the above questions, but now just for the question number (3): looking for new hierarchy of integrable systems, which is of fundamental importance in the study of Hamiltonian integrable systems [4-7, 9-12]. A new hierarchy of integrable systems of $1+2$ dimensions with its Hamiltonian form is proposed in the next section.

\section{A new hierarchy of integrable system of $1+2$ dimensions}

In the past decades there is a growing interest in the $1+2$-dimensional systems that involve two spatial variables $x, y$ and one temporal variable $t$. The theory on $1+2$ dimensional systems is much more complicated than the one on $1+1$-dimensional systems, which involves only $x$ and $t$. It was known that $1+1$-dimensional integrable systems possess a remarkable rich algebraic structure, such as the existence of infinite number of symmetries and conserved densities, the existence of bi-Hamiltonian structures and so on. A certain operator $\phi$, called recursion operator, plays a central role in investigating the above algebraic properties (see, e.g., Magri and Morosi [7], Zhang [14]). One could naturally expect that such operators would exist also in 1+2-dimensional case. However, in [13] Zakharov and Konopel'chenko announced that such operators are merely onedimensional matter, and, moreover, there does not exist a recursion operator in $1+2$ dimensional case. A major step was made by Fokas and Santini in a series of papers (see Fokas and Santini $[1,2,8])$. They successfully developed a unified theory on recursion operators of both $1+1$ - and $1+2$-dimensional systems. In their construction of recursion operators the Dirac function $\delta(x-y)$ is frequently used. Since the Dirac function is a kind of distributions (generalization functions), it is natural to expect for developing a pure algebraic approach to the theory of recursion operators. In [7], Magri and Morosi proposed such an algebraic approach. They constructed a recursion operator of the famous KP hierarchy. However their recursion scheme is not direct. In order to reach the KP hierarchy they had to use several initial values $\phi_{j}$ and then making linear combination of $\phi_{i} \phi_{j}$.

Fokas and $\mathrm{Tu}$ [3] (see, also [11]) propose a new algebraic recursion scheme for generating integrable systems of $1+2$ dimensions. Their method was based on the theory of pseudodifferential operators. The aim of this section is to propose another hierarchy of 
$1+2$-dimensional systems by following the residue approach of Fokas and $\mathrm{Tu}[3]$ and $\mathrm{Tu}$ et al. [11]. The first equation (after a simple reduction) in this hierarchy reads

$$
q_{t}=i\left(q_{x}-q_{y}-q^{2}+2|q|^{2}\right)_{x}
$$

Let $A$ be an associative algebra over the field $K=C$ or $R$, and let $\partial: A \rightarrow A$ be a derivation, that is,

$$
\partial(\alpha f+\beta g)=\alpha \partial f+\beta \partial g, \quad \partial(f g)=f(\partial g)+(\partial f) g
$$

where $\alpha, \beta \in k$ and $f, g \in A$. Following the notation of $[3,11]$, we form an associative algebra $A[\xi]$, which consists of all pseudodifferential operators $\sum_{-\infty}^{N} a_{i} \xi^{i}$, where the coefficients $a_{i}^{\prime}$ s are taken from $A: a_{i} \in A$, and $\xi$ stands for an operator defined by

$$
\xi f=f \xi+(\partial f), \quad f \in A .
$$

The following operator $R: A[\xi] \rightarrow A$,

$$
R\left(\sum a_{i} \xi^{i}\right)=a_{-1}
$$

is called the residue operator due to the similarity with the residue in theory of complex analysis.

The technique proposed by Fokas and Tu [3] for generating the KP and DS hierarchies can be briefly described as follows.

First we fix a matrix operator $U=U(\lambda, u) \in A[\xi]$, which depends on a parameter $\lambda$ and a vector function $u=\left(u_{1}, \ldots, u_{p}\right)^{T}$.

Second, solve the equation

$$
V_{x}=[U, V]
$$

for $V=\sum V_{n} \lambda^{-n}$. By solving the recursion relation among $V_{n}$ 's we will obtain a recursion relation among

$$
g^{(n)} \equiv\left(g_{1}^{(n)}, \ldots, g_{p}(n)\right)^{T}
$$

where $g_{i}^{(n)}$ comes from the expansion

$$
\left\langle V, \frac{\partial U}{\partial u_{i}}\right\rangle=\sum_{n} g_{i}^{(n)} \lambda^{-n}
$$


8 A new hierarchy 1+2-dimensional integrable system

here $\langle\cdot, \cdot\rangle$ is defined as

$$
\langle a, b\rangle=\operatorname{tr}(R(a b))
$$

Third, we try to find an operator $\mathbf{J}$, and then form the hierarchy

$$
u_{t_{n}}=J g^{(n)}
$$

In the case of KP and DS hierarchies, the operator $\mathbf{J}$ is the same as used in the corresponding 1+1-dimensional case.

Following the above approach we are able to suggest the following new hierarchy.

Levi (see $[9,10])$ proposed a hierarchy starting from the matrix

$$
\left[\begin{array}{cc}
-q & \mu q \\
-\mu & \mu^{2}+r
\end{array}\right]
$$

It is not difficult to show that the above matrix is similar to the matrix

$$
\left[\begin{array}{cc}
0 & q \\
r & \lambda+r-q
\end{array}\right]
$$

Thus the same hierarchy will be obtained if one starts from the above matrix. Motivated by this hierarchy we try to generate a similar one of $1+2$ dimensions. Let us begin with the following operator:

$$
\left[\begin{array}{cc}
0 & q \\
r & \lambda+\xi+r-q
\end{array}\right]
$$

Substituting the formula (2.12) and

$$
V=\left[\begin{array}{ll}
A & B \\
C & D
\end{array}\right]=\sum_{n \geq 0} V_{n} \lambda^{-n}, \quad V_{n}=\left[\begin{array}{cc}
A_{n} & B_{n} \\
C_{n} & D_{n}
\end{array}\right]
$$

into the following equation:

$$
V_{x}=[U, V]
$$

yields

$$
\begin{gathered}
A_{n x}=q C_{n}-B_{n} r, \quad D_{n x}-D_{n y}+\left[q-r, D_{n}\right]=r B_{n}-C_{n} q, \\
B_{n+1}=-B_{n x}+q D_{n}-A_{n} q+B_{n}(q-r)-B_{n} \xi \\
C_{n+1}=C_{n x}+D_{n} r-r A_{n}+(q-r) C_{n}-\xi C_{n} .
\end{gathered}
$$

From the above recursion relation we see that $C_{0}=B_{0}=0$. Now we take the following initial operators:

$$
A_{0}=\alpha \xi^{-1}, \quad D_{0}=0
$$


It is easy to calculate the subsequent $A_{n}, B_{n}, C_{n}$, and $D_{n}$ as follows:

$$
\begin{gathered}
B_{1}=\alpha\left(-q \xi^{-1}+q_{y} \xi^{-2}\right)+O\left(\xi^{-3}\right), \\
C_{1}=\alpha\left(-r \xi^{-1}\right), \quad A_{1 x}=O\left(\xi^{-2}\right), \quad D_{1}=0, \\
B_{2}=\alpha\left(q+\left(q_{x}-q_{y}-q^{2}+q r\right) \xi^{-1}\right)+O\left(\xi^{-2}\right), \\
C_{2}=\alpha\left(r+\left(r_{y}-r_{x}+r^{2}-q r\right) \xi^{-1}\right)+O\left(\xi^{-2}\right), \\
A_{2 x}=\alpha\left(-q r+\left((q r)_{y}-(q r)_{x}\right) \xi^{-1}\right)+O\left(\xi^{-2}\right), \quad D_{2}=\alpha\left((q r) \xi^{-1}\right)+O\left(\xi^{-2}\right) .
\end{gathered}
$$

Since in present case we have

$$
\left\langle V, U_{q}\right\rangle=C-D, \quad\left\langle V, U_{r}\right\rangle=B+D,
$$

and since the operator $\mathbf{J}$ in the $1+1$-dimensional case is

$$
J=\left[\begin{array}{cc}
0 & \partial_{x} \\
\partial_{x} & 0
\end{array}\right],
$$

we obtain the following hierarchy:

$$
q_{t_{n}}=R\left(B_{n x}+D_{n x}\right), \quad r_{t_{n}}=R\left(C_{n x}-D_{n x}\right) .
$$

The first nontrivial pair of equations in this hierarchy are

$$
\begin{gathered}
q_{t_{1}}=\alpha q_{x}, \quad r_{t_{1}}=\alpha r_{x}, \\
q_{t_{2}}=\alpha\left(q_{x}-q_{y}-q^{2}+2 q r\right)_{x}, \quad r_{t_{2}}=-\alpha\left(r_{x}-q_{y}-r^{2}+2 q r\right)_{x} .
\end{gathered}
$$

We observe that (2.21) reduces to the LMN equation when $q_{y}=0$. Equation (2.21) admits a reduction $\alpha=i, q=r *(r *$ is the complex conjugate of $r)$,

$$
q_{t}=i\left(q_{x}-q_{y}-q^{2}+2|q|^{2}\right)_{x} .
$$

By applying the trace identity as Tu did in the case of KP and DS hierarchies [9], we can write the above 2D LNM hierarchy in its Hamiltonian form:

$$
u_{t_{n}}=J \frac{\delta H_{n}}{\delta u}
$$

where

$$
H_{n}=-\frac{D_{n+1}}{n}
$$

We thus complete this paper.

\section{Acknowledgment}

The authors extend their thanks to Professor Roman Andrushkiw for his useful discussion. 


\section{References}

[1] A. S. Fokas and P. M. Santini, The recursion operator of the Kadomtsev-Petviashvili equation and the squared eigenfunctions of the Schrödinger operator, Studies in Applied Mathematics 75 (1986), no. $2,179-185$.

[2] __ Recursion operators and bi-Hamiltonian structures in multidimensions. II, Communications in Mathematical Physics 116 (1988), no. 3, 449-474.

[3] A. S. Fokas and G. Tu, An algebraic recursion scheme for KP and DS hierarchies, Tech. Rep., Clarkson University, New York, 1990.

[4] B. Fuchssteiner, Coupling of completely integrable systems: the perturbation bundle, Applications of Analytic and Geometric Methods to Nonlinear Differential Equations (Exeter, 1992), NATO Adv. Sci. Inst. Ser. C Math. Phys. Sci., vol. 413, Kluwer Academic, Dordrecht, 1993, pp. 125-138.

[5] F. K. Guo, A hierarchy of integrable Hamiltonian equations, Acta Mathematicae Applicatae Sinica 23 (2000), no. 2, 181-187.

[6] W. X. Ma and B. Fuchssteiner, Integrable theory of the perturbation equations, Chaos, Solitons and Fractals 7 (1996), no. 8, 1227-1250.

[7] F. Magri and C. Morosi, Old and new results on recursion operators: an algebraic approach to KP equation, Topics in Soliton Theory and Exactly Solvable Nonlinear Equations (Oberwolfach, 1986) (M. Ablowitz, B. Fuchssteiner, and M. Kruskal, eds.), World Scientific, Singapore, 1987, pp. 78-96.

[8] P. M. Santini and A. S. Fokas, Recursion operators and bi-Hamiltonian structures in multidimensions. I, Communications in Mathematical Physics 115 (1988), no. 3, 375-419.

[9] G. Tu, The trace identity, a powerful tool for constructing the Hamiltonian structure of integrable systems, Journal of Mathematical Physics 30 (1989), no. 2, 330-338.

[10] _ A trace identity and its applications to the theory of discrete integrable systems, Journal of Physics. A. Mathematical and General 23 (1990), no. 17, 3903-3922.

[11] G. Tu, R. I. Andrushkiw, and X. Huang, A trace identity and its application to integrable systems of $1+2$ dimensions, Journal of Mathematical Physics 32 (1991), no. 7, 1900-1907.

[12] G. Tu and X. Huang, From Newton's law to generalized Hamiltonian systems I: some results on linear skew-symmetric operator, Journal of Yangzhou Polytechnic University 9 (2005), no. 2, 3646.

[13] V. E. Zakharov and B. G. Konopel'chenko, On the theory of recursion operator, Communications in Mathematical Physics 94 (1984), no. 4, 483-509.

[14] Y. F. Zhang, A subalgebra of a Lie algebra and two types of associated loop algebras, Acta Mathematica Sinica 48 (2005), no. 1, 141-152.

Xuncheng Huang: College of International Exchange, Yangzhou Polytechnic University,

Yangzhou, Jiangsu 225002, China

E-mail address: xunchengh@yzpc.edu.cn

Guizhang Tu: Bloomberg L. P., NY 10019, USA

E-mail address: tugz@yahoo.com 


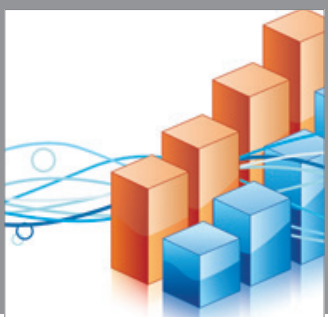

Advances in

Operations Research

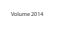

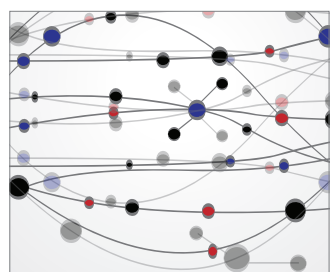

\section{The Scientific} World Journal
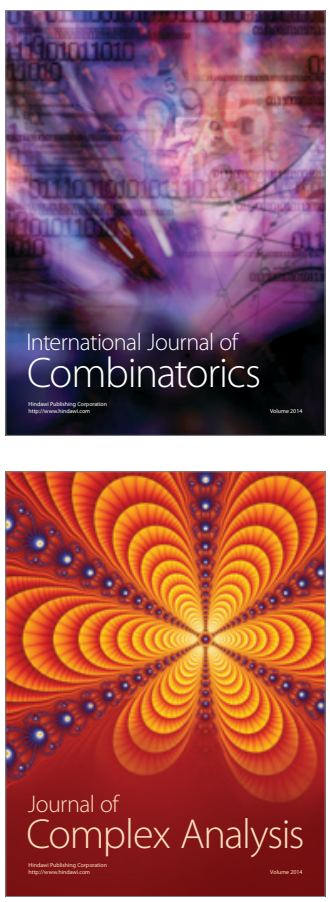

International Journal of

Mathematics and

Mathematical

Sciences
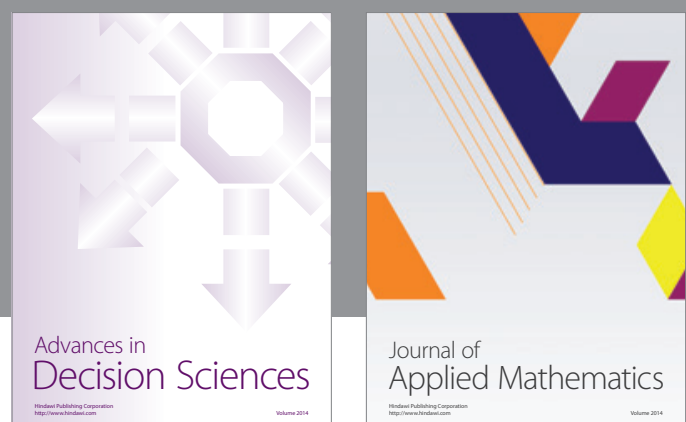

Journal of

Applied Mathematics
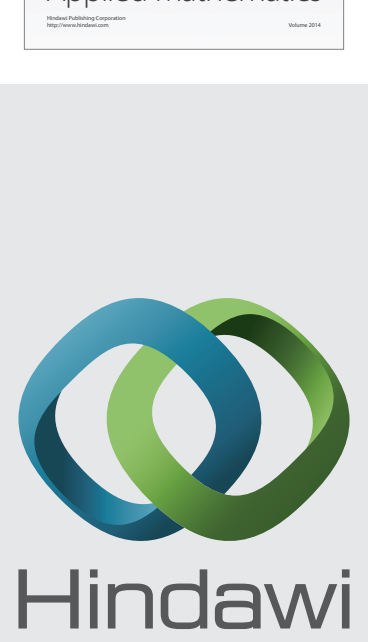

Submit your manuscripts at http://www.hindawi.com
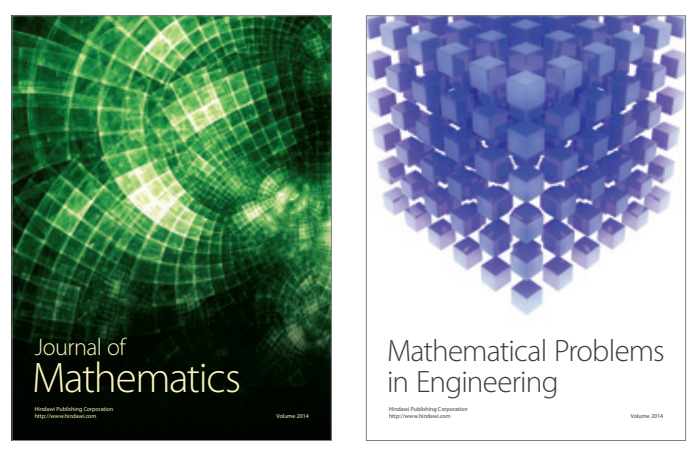

Mathematical Problems in Engineering
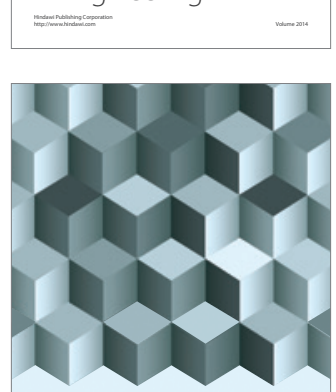

Journal of

Function Spaces
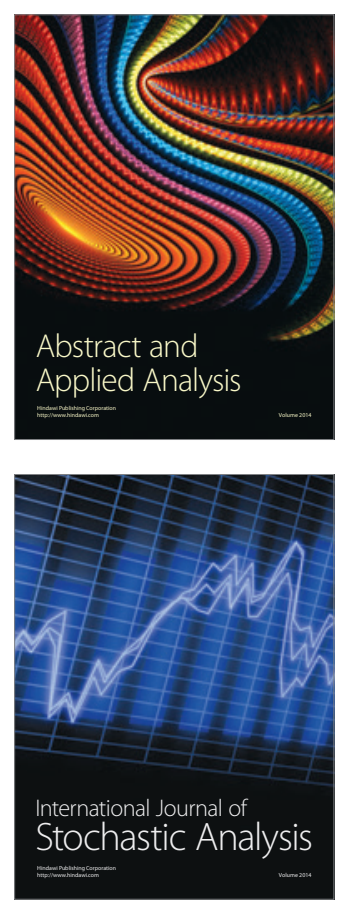

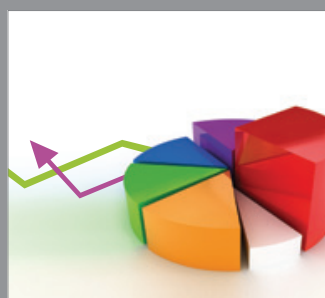

ournal of

Probability and Statistics

Promensencen
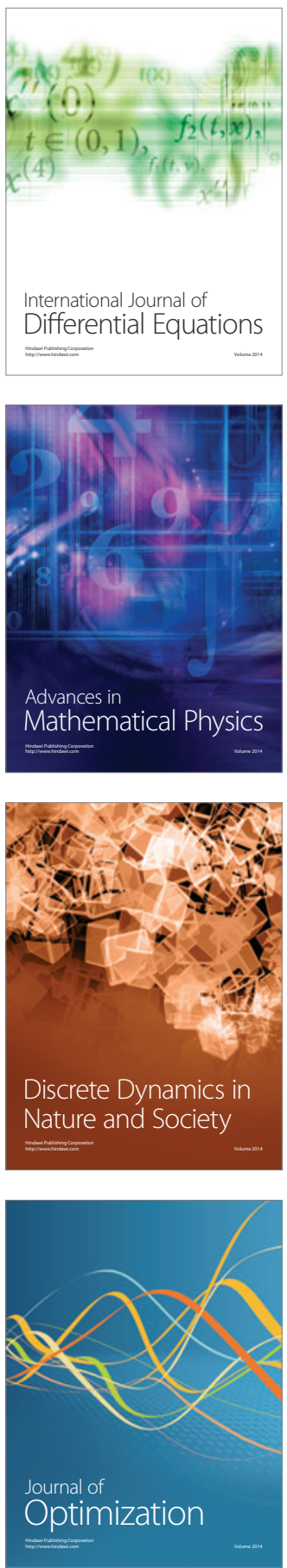